\title{
The involvement of neuronal nitric oxide synthase in antiepileptic action of alpha- asarone on pentylenetetrazol molding rats
}

\author{
Jing Su ${ }^{\mathrm{a}}$, Wenting Zhu ${ }^{\mathrm{b}}$, Jing Liu ${ }^{\mathrm{c}}$, Jian Yin ${ }^{\mathrm{a}}$, Wei Qin ${ }^{\mathrm{a}}$ and Changbin Jiang ${ }^{\mathrm{d}, *}$ \\ ${ }^{a}$ Department of Neurosurgery and Epilepsy Center, the Second Affiliated Hospital of Dalian Medical \\ University, Dalian, China \\ ${ }^{b}$ Dalian Medical University, Dalian, China \\ ${ }^{c}$ Regenerative Medicine Centre, the First Affiliated Hospital of Dalian Medical University, Dalian, \\ China \\ ${ }^{d}$ Department of Neurology, the First Affiliated Hospital of Dalian Medical University, Dalian, China
}

\begin{abstract}
The aim of the present study was to research the role of nitric oxide (NO) as a mediator of alpha $(\alpha)$-asarone effect at the pentylenetetrazol (PTZ)-induced epileptiform discharge in rat. $\alpha$-Asarone that was injected intraperitoneally twenty minutes before PTZ injection suppressed the clonic discharge effectively and the significant actions lasted for 30 min with no change of clonic amplitude. Administration of $\alpha$-asarone did not influence interictal discharge. Four kinds of NO regulators were administered, including non-selective NG-nitro-L-arginine methyl ester (L-NAME), selective neuronal nitric oxide synthase (nNOS) inhibitor, 7-nitroindazole (7-NI), inducible nitric oxide synthase (iNOS) inhibitor, aminoguanidine (AG) and NO substrate, L-arginine (ARG) and their influence on the actions of $\alpha$-asarone were studied, and all of the regulators were administered fifteen minutes before $\alpha$-asarone injection. L-NAME and 7-NI reversed the anticlonic activity of $\alpha$-asarone, and a significant increase of clonic activity was induced by L-NAME later in L-NAME +. $\alpha$-asarone + PTZ group. There were no significant differences between $\mathrm{AG}+\alpha$-asarone + PTZ and $\alpha$-asarone + PTZ group. L-ARG played a dual role in this study. It aggravated clonic discharge in the early stage but relieved interictal discharge in the late stage compared with PTZ group alone, and the beneficial effect of $\alpha$-asarone was also reversed. All the above results suggest that nNOS/NO pathway mediates the anticonvulsant effect of $\alpha$-asarone, and NO played a biphasic role in PTZ modeling process, while iNOS was unrelated to the inhibition effect of $\alpha$-asarone on PTZ induced epileptiform activity.
\end{abstract}

Keywords: Alpha-asarone, epileptiform activity, nitric oxide synthase, pentylenetetrazol

\section{Introduction}

Acorus calamus Linn (AC) has been traditionally used in oriental prescriptions for hundreds of years. Roots and rhizomes of $\mathrm{AC}$ were used as orient traditional medicine for the treatment of epilepsy and memory disorders [1]. The calamus oil of AC predominantly contain asarone [2]. Alpha ( $\alpha$ )-asarone, the trans isomers of the alkenylbenzene 1-propenyl-2, 4, 5-trimethoxybenzene has antioxidant and

\footnotetext{
${ }^{*}$ Corresponding author: Changbin Jiang, Department of Neurology, the First Affiliated Hospital of Dalian Medical University, Dalian, China. Tel.: 13555988100; Fax: 086-411-87630449; E-mail: 13555988100@126.com.
} 
neuroprotective role [1]. Jungsook et al. [3] found that $\alpha$-asarone protected neurons from the excitotoxicity induced by glutamate (Glu) or N-methyl-D-aspartate (NMDA) in primary cultured rat neurons. Others found that $\alpha$-asarone exerted annticonvulsant effect in both PTZ and kainate models in vitro [4].

Redox reactions involve in epileptic attacks, and the pathological process of epilepsy is not clear [5]. Nitric oxide (NO) is a universal neuronal messenger in the brain, in the pathophysiology of seizure attacks [6]. NO is a gaseous free radical and functions as a modulator of neurotransmitters in the brain. $\mathrm{L}$-arginine is the substrate for NO synthesis and three kinds of nitric oxide synthase (NOS) isoforms participate in NO generation which are endothelial NOS (eNOS), inducible NOS (iNOS), and neuronal NOS (nNOS) [7]. NO is regarded as important pathogenetic factor in the mechanisms underlying seizure induction and progression [8].

However, the mechanism for the beneficial action of $\alpha$-asarone on epilepsy remains unclear. This study was to research the actions of $\alpha$-asarone on the NO generation during model epileptiform seizures. There have been no published information available about the role of $\mathrm{NO}$ in the beneficial action of $\alpha$-asarone on epileptic discharge. Thus, we studied the possible correlation of NOS/NO with the effects of $\alpha$-asarone on PTZ-induced epileptiform ECoG discharge in the rat, using nonspecific NOS inhibitor L-NAME(inhibiting both eNOS and nNOS ), selective iNOS inhibitor AG, selective nNOS inhibitor 7-NI and NO substrate, L-ARG.

\section{Results}

ECoG of rats that were not given any treatment was regarded as blank control (Figure 1A). As expected, Intraperitoneal injection of physiological saline and mixed solvent (DMSO/polyethylene glycol $3001: 1, \mathrm{v} / \mathrm{v}$ ) (Figure 1B) did not influence the frequency or amplitude of ECoG discharge compared with the control group (Figure 1A) in non-PTZ treated rats. Injection of $80 \mathrm{mg} / \mathrm{kg} \alpha$-asarone in non-PTZ injected animals caused no aberrant epileptiform ECoG discharge (Figure 1C). Baseline waves of each rat were recorded before the application of chemicals, and no spontaneous seizures have been confirmed. Intraperitoneal administration of PTZ $(50 \mathrm{mg} / \mathrm{kg})$ induced epileptiform activity in all the experimental animals characterized by clonic activity and interictal discharge, with or without tonic/clonic seizures. These abnormal discharges began within 5 min after PTZ administration and lasted for no less than $1.5-2 \mathrm{~h}$.

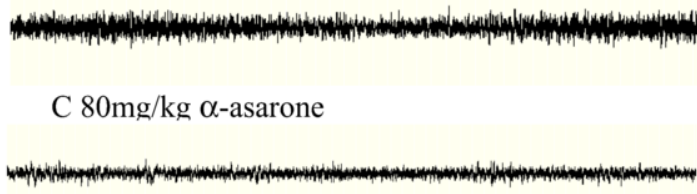

Fig. 1. Representative ECoG recordings of Control baseline, mixed solvent and $80 \mathrm{mg} / \mathrm{kg} \alpha$-asarone. 


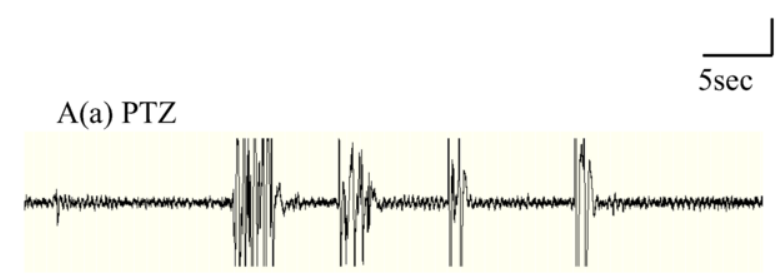
$100 \mu \mathrm{V}$
(b) $\alpha$-asarone + PTZ

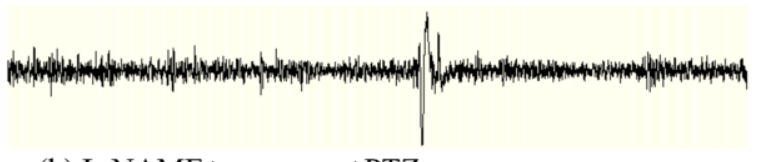

B(a) PTZ

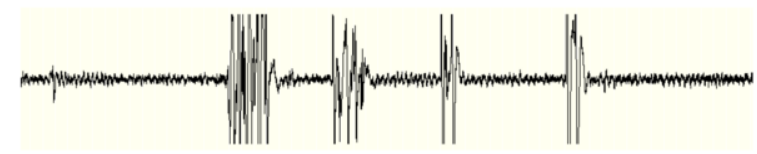

(b) L-NAME $+\alpha$-asarone + PTZ

C(a) PTZ

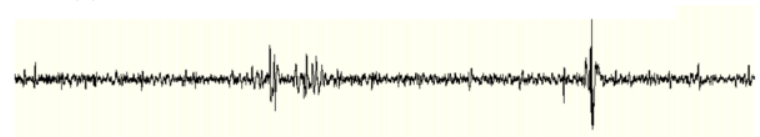

D(a) PTZ

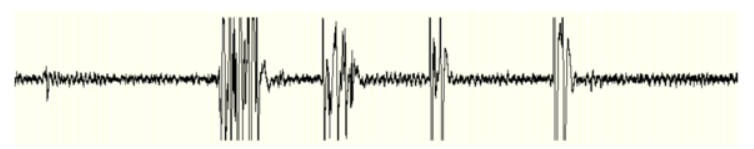

(b) $7-\mathrm{NI}+\alpha$-asarone $+\mathrm{PTZ}$

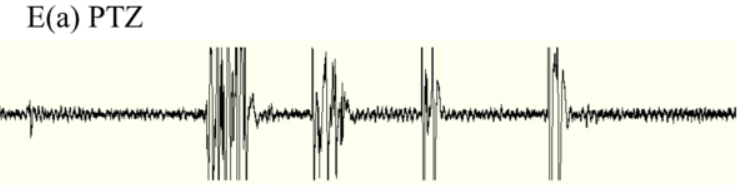

F(a) PTZ

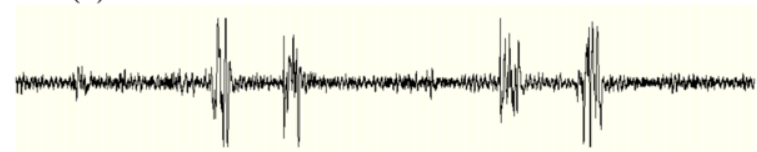

G(a) PTZ

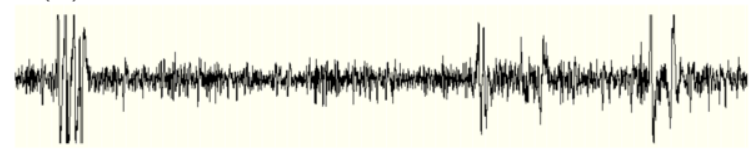

(b) L-NAME $+\alpha$-asarone + PTZ
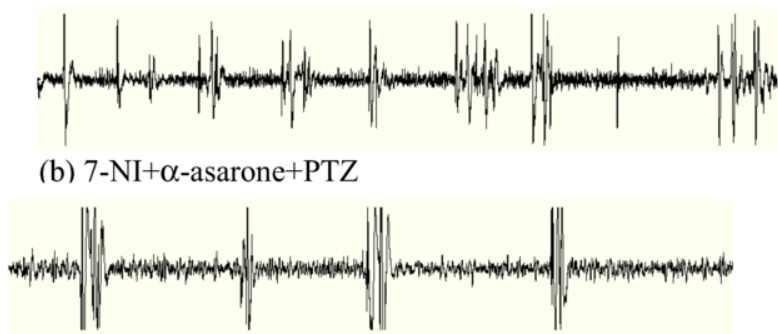

(b) $\mathrm{AG}+\alpha$-asarone $+\mathrm{PTZ}$

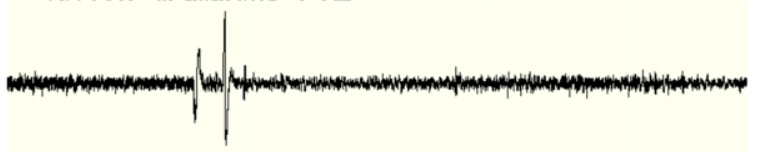

(b) L-ARG $+\alpha$-asarone $+\mathrm{PTZ}$

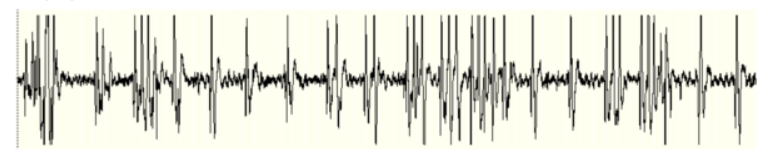

(b) $\mathrm{L}$-ARG $+\alpha$-asarone $+\mathrm{PTZ}$

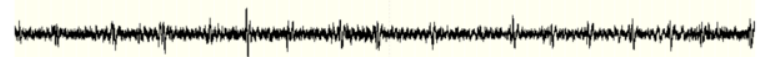

Fig. 2. (A) $\alpha$ - Asarone significantly decreased the clonic activity compared with PTZ group alone. (B) L-NAME reversed the anticlonic activity of $\alpha$-asarone. (C) L-NAME significantly increased the clonic activity. (D) 7-NI reversed the anticlonic activity of $\alpha$-asarone. (E) AG significantly decreased the clonic activity compared to that of PTZ group (F) L-ARG significantly increased the clonic activity $(\mathrm{G})$ L-ARG significantly decreased the interictal discharge.

Alpha- asarone, which is $80 \mathrm{mg} / \mathrm{kg}$, was injected $20 \mathrm{~min}$ before PTZ molding and significantly reduced the mean frequency of clonic discharge to $28.5 \pm 15.9$ clonic/min in the $50 \mathrm{~min}$ after PTZ molding (i.p.) (Figures $2 \mathrm{~A}$ and 3). The significant effects lasted for $30 \mathrm{~min}$ (Figure 3) with no change of clonic amplitude. Administration of $\alpha$-asarone did not influence the frequency of interictal discharge (Figure 4). L-NAME (60 mg/kg, i.p.) was injected $15 \mathrm{~min}$ before $\alpha$-asarone administration and reversed the anticlonic activity of $\alpha$-asarone compared with $\alpha$-asarone + PTZ group (Figures 2B and 3), and a significant increase of clonic activity was induced 70 min after PTZ injection in L-NAME $+\alpha-$ asarone $+\mathrm{PTZ}$ group (Figure 3). The mean frequency of clonic discharge was $78.8 \pm 33.8 \mathrm{clonic} / \mathrm{min}$ in the 80 min after PTZ molding in L-NAME group (Figures $2 \mathrm{C}$ and 3). While there were no significant 
changes of clonic amplitude. 7-NI (40 mg/kg, i.p.) also reversed the anticlonic activity of $\alpha$-asarone in 7 -NI $+\alpha$-asarone + PTZ group (Figure 3). The mean frequency of clonic discharge was $74.7 \pm 32.8$ clonic/min in the $50 \mathrm{~min}$ after PTZ injection (Figure 2D). There were no significant changes of clonic amplitude in 7-NI group. In the AG $(100 \mathrm{mg} / \mathrm{kg}$, i.p. $)+\alpha$-asarone + PTZ group, the clonic discharge was also reduced significantly compared with that of PTZ group, and there were no significant differences between $\mathrm{AG}+\alpha$-asarone $+\mathrm{PTZ}$ and $\alpha$-asarone + PTZ group (Figure 3 ). The mean frequency of clonic discharge was $24.7 \pm 12.4$ clonic/min in the 50 min after PTZ molding in AG group (Figures 2E and 3). There were no significant changes of clonic amplitude in AG group. All the NOS inhibitors did not influence either the amplitude or the frequency of interictal discharge (Figure 4). In the L-ARG $(500 \mathrm{mg} / \mathrm{kg}$, i.p.) $+\alpha$-asarone + PTZ group, contradictory results occurred. The clonic discharge was temporally increased in the 20min after PTZ injection compared with PTZ group and the anticlonic activity of $\alpha$-asarone was reversed (Figure 3 ) compared with $\alpha$-asarone + PTZ group. The mean frequency of clonic discharge was $194.3 \pm 76.6 \mathrm{clonic} / \mathrm{min}$ in the $20 \mathrm{~min}$ after PTZ molding in L-ARG group (Figure $2 \mathrm{~F}$ ). Whereas the mean frequency of interictal discharge significantly decreased in the $60 \mathrm{~min}$ after PTZ injection (Figure $2 \mathrm{G}$ ) and lasted for $20 \mathrm{~min}$ compared with PTZ group. There were no significant changes of clonic amplitude in L-ARG group.

\section{Discussion}

Epilepsy is a common disease in the central nervous system, and the population prevalence rate is close to $1 \%$ [9]. Some researchers suggest that oxidative stress involves in the occurrence and development of epilepsy, resulting in aberrant structural changes of cellular proteins, membrane lipids, DNA and RNA [10]. Peroxidation of lipid has been enhanced in several animal models of epilepsy

The interactions of $\alpha$-asarone and No on the clonic frequency

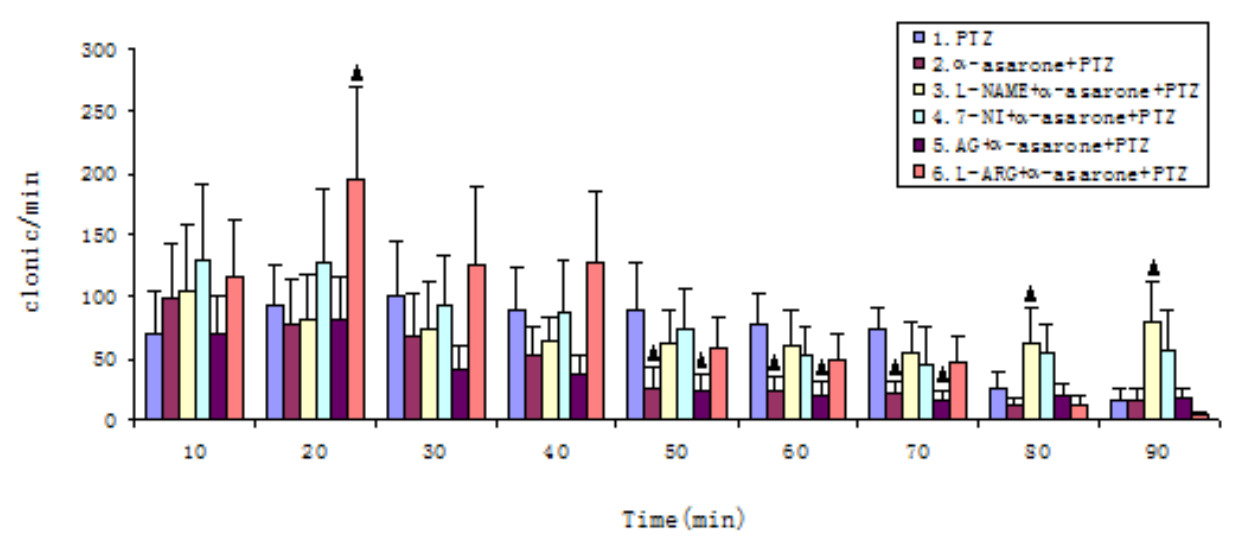

Fig. 3. $\alpha$ - Asarone significantly decreased the clonic activity in the $50 \mathrm{~min}$ after PTZ injection and the significant effects lasted for $30 \mathrm{~min}$. L-NAME reversed the anticlonic activity of $\alpha$-asarone compared with $\alpha$-asarone + PTZ group, and a significant increase of clonic activity was induced $70 \mathrm{~min}$ after PTZ injection. 7-NI also reversed the anticlonic activity of $\alpha$-asarone. In the AG $+\alpha$-asarone + PTZ group, the clonic activity was decreased significantly compared to that in PTZ group. In the L-ARG + aasarone $+\mathrm{PTZ}$ group, the clonic activity was temporally increased in the $20 \mathrm{~min}$ after PTZ injection compared with PTZ group alone and the anticlonic activity of $\alpha$-asarone was reversed compared with $\alpha$-asarone + PTZ group. $\mathbf{\Delta} \mathrm{p}<0.05$ (When compared with PTZ group). 


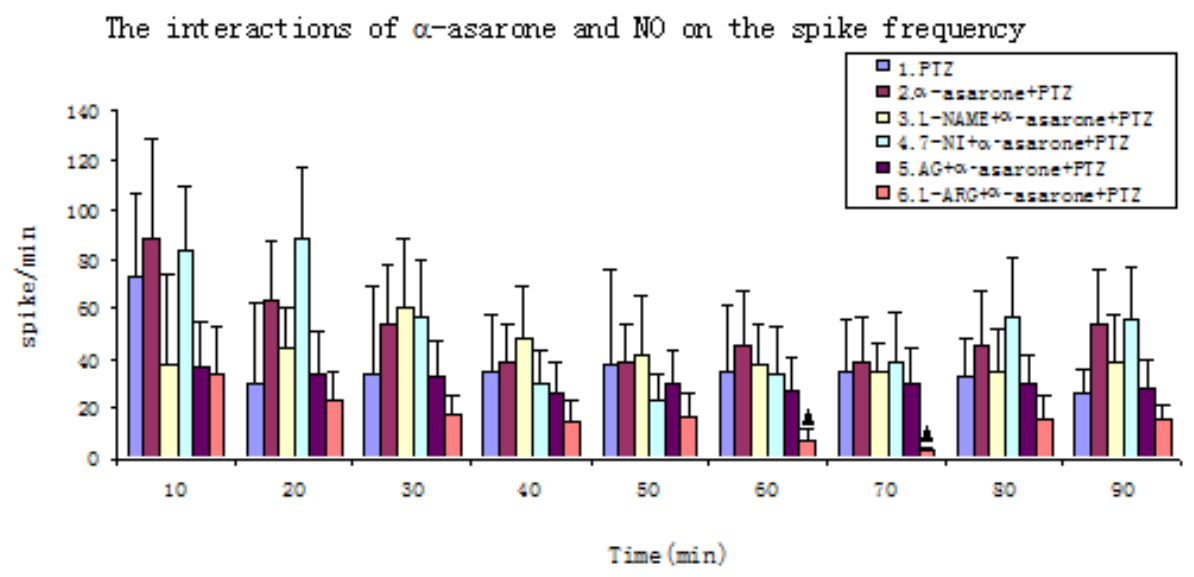

Fig.4.Administration of $\alpha$-asarone, L-NAME, 7-NI and AG did not influence the frequency of interictal discharge. In the L-ARG $+\alpha$-asarone + PTZ group, the mean frequency of interictal discharge significantly decreased in the $60 \mathrm{~min}$ after PTZ injection and lasted for 20min compared with PTZ group. $\mathbf{\Delta} \mathrm{p}<0.05$ (When compared with PTZ group).

[11]. These experimental investigations have reported that seizures cause oxidative stress, and similar results are confirmed in research of epileptic patients [5], as well as others [12]. NO involve in many physiological and pathophysiological processes in the central nervous system, including the modulation of neuronal plasticity, cerebral blood-flow, cognitive and behavioral functions, as well as, its influence on diseases such as ischemia and epilepsy [13]. A comprehensive understanding of the role of NO might be in favor of the development of effective antiepileptic treatment [14].

$\alpha$-Asarone was reported to influence redox reaction in noise stress [15]. $\alpha$-asarone was suggested to decrease the incidence and duration of tonic seizures induced by maximal electroshock(MES) [16] and by pentylenetetrazole [17], at doses of 20 and $50 \mathrm{mg} / \mathrm{kg}$, respectively. Chen et al. [18] reported that application of $\alpha$-asarone $(50-200 \mathrm{mg} / \mathrm{kg}$, p.o.) repeated treatment effectively suppressed maximal electroshock seizure and PTZ-induced seizures, and that a single acute administration of $\alpha$-asarone (50$200 \mathrm{mg} / \mathrm{kg}$, p.o.) exhibited anticonvulsant activity in MES and PTZ kindling animal models. We used a dose of $80 \mathrm{mg} / \mathrm{kg} \alpha$-asarone to produce maximum antiepileptic action in this research. Reports previously showed that $\alpha$-asarone reduced the frequency of PTZ-induced epileptiform activity in rat without concerning the involvement of NOS/NO pathway [18]. For this reason, we studied the role of nitric oxide in the effects of $\alpha$-asarone on PTZ-kindled epileptiform ECoG discharge in the rat. In order to get both attack and interictal aberrance in the model, we used moderate dose of PTZ in our study which is $50 \mathrm{mg} / \mathrm{kg}$.

NO was suggested to be an endogenous anticonvulsant in previous report [19], while others suggest a proconvulsant role for NO [20,21]. Mehmet et al. [22] reported that L-NAME $(100 \mathrm{mg} / \mathrm{kg}) \mathrm{did}$ not affect both of the frequency and amplitude of penicillin induced epileptiform discharge in rats. It was suggested that L-NAME was able to weaken the beneficial action of some conventional anticonvulsive drugs [23]. In the present research, L-NAME reversed the anticlonic activity of $\alpha$-asarone and a significant increase of clonic activity was induced 70 min after PTZ injection in L-NAME $+\alpha$-asarone + PTZ group (Figure 3). This result supports the previous findings indirectly that suggested the suppression of L-NAME upon protective actions of some chemicals which have anticonvulsive properties [23-25]. Our results showed that injection of 7-NI ( $40 \mathrm{mg} / \mathrm{kg}$, i.p. $)$ also reversed the anticlonic effect of $\alpha$-asarone in 7-NI $+\alpha$-asarone + PTZ group (Figure 3).Considering the above results in the present 
study, It seemed that nNOS involved in mediating the beneficial effect of $\alpha$-asarone on PTZ-induced epilepsy model. This finding is consistent with that of previous researchers whose investigation demonstrated proconvulsant effects of 7-NI $[19,26]$, but is incosistent with reports which have demonstrated the protective effect of 7-NI $[24,25,27]$. As to the deterioration effect of L-NAME in the action effect of $\alpha$-asarone, it can be speculated that either the endothelial-NOS activity involve in the proconvulsant action of $\alpha$-asarone or activating effect of L-NAME on the proconvulsant action of $\alpha$-asarone might be its own nonspecific action without correlations with endothelial-NOS action in the central nervous system. If this is not the case, other pharmacological mechanisms of L-NAME and $\alpha$-asarone might be involved in, unrelated to the NOS/NO pathway in the brain. There were no significant differences between AG $+\alpha$-asarone + PTZ and $\alpha$-asarone + PTZ group. These results may suggest that iNOS is unrelated to the inhibition effect of $\alpha$-asarone on PTZ induced epileptiform activity. These results are also consistent with previous reports revealing the action of constitutive NO (eNOS and nNOS) pathway involving in the anticonvulsant mechanisms [28]. While it is inconsistent with previous report that demonstrated that iNOS mediates the PTZ-induced convulsant status. In some reports, L-ARG was suggested to aggravate the severity of experimental epileptic attacks and NO was speculated to have a proconvulsive effect [29]. Previous experimental investigations have revealed that inhibition of NO suppresses convulsion [30]. De Vasconcelos et al. [31] reported that NG-nitro-Larginine enhanced severity of seizures and L-ARG aggravated hurt of convulsion. Direct injection of NO (330-800 $\mu \mathrm{mol})$ into the rat cortex resulted in brief tonic convulsive attacks [32] and 3morpholinylsydnoneimine (SIN-1), a NO substrate, induced convulsion in DBA/2 mice and genetically epilepsy-prone rats[20].There are also some examples of opposition. L-ARG (500 mg/kg, i.p.) effectively decreased the mean frequency of epileptiform discharge without influencing amplitude in the penicillin kindling rats $[25,33]$. It was reported that L-ARG strengthened the antiepileptic effect of ghrelin through an observation of reduction of epileptic discharge compared with both of L-ARG [33] and ghrelin group alone [34]. Rundfeldt and his team [35] proposed that NO has dual roles either as a convulsant or an anticonvulsant in the same animal experiment depending on the dose applied. Analogous report was that NO increase may play different actions depending on whether this change occurs before or after the beginning of the seizure attack [36]. In this study, L-ARG (500 mg/kg, i.p.) played a dual role. It aggravated clonic activity in the early stage but relieved interictal discharge in late stage, and the beneficial effect of $\alpha$-asarone was reversed. These results indicated that NO might have biphasic effect during PTZ induced epilepsy progression. Some researchers viewed that NO is a consequent product after the occurrence of epilepsy kindling in experimental models [37]. In animal experimental investigations, the researchers found the apparent upregulation of extracellular NO level and a manifest increase of NOS immunoreactivity in cortex after kindling [38], including contralateral temporal lobe structures [21]. It was found that the aberrant development of the fibers of nNOS neurons exists in the human hippocampus with hippocampal sclerosis [39]. In GABA antagonists kindling animal seizures, the application of L-ARG induced an excessive increase level of NO, which may result in a hyperexcitability state via the production of cGMP [40]. It was viewed that the hyperactivity of NO neurons might lead to abnormal cortical excitability for NO promote neurotransmitter release, and NO may subsequently decrease the activity of the inhibitory GABAergic receptors [41]. However, NO was suggested to have adaptative neuroprotective actions for it can suppress an overactivity of NMDA receptors $[26,38]$. This may explain the conflict role of L-ARG in the two phases. Therefore, we might assume that excessive NO induced abnormal cortical excitability, which triggered a subsequent inhibitory effect through some feedback mechanism with lots of cascades inside. These conflicting results might be due to the effect of L-ARG application, which interfered the balance of NO before modeling. All together, our data suggested the involvement of nNOS/NO mechanism in the antiepileptic action 
of $\alpha$-asarone, and NO played biphasic role in PTZ modeling process, which exhibit proconvulsive effect in early stage and anticonvulsive effect in late stage. For all test compounds, the action effect did not show exactly the same in the two kinds of aberrant epileptiform activities (clonic activity and interictal discharge). It may be implied that there were some differences in the formation mechanism between two kinds of epileptiform discharge.

\section{Conclusion}

We confirmed that $\alpha$-asarone significantly inhibited clonic activition in PTZ induced epilepsy model. The research results showed that L-NAME reversed the anticlonic activity of $\alpha$-asarone and a significant increase of clonic activity was induced 70 min after PTZ injection in L-NAME $+\alpha$-asarone + PTZ group. 7-NI also reversed the anticlonic activity of $\alpha$-asarone in 7-NI $+\alpha$-asarone + PTZ group. There were no significant differences between AG $+\alpha$-asarone + PTZ and $\alpha$-asarone + PTZ group. L-ARG played a dual role. It aggravated clonic activity in the early stage but relieved interictal discharge in late stage, and the beneficial effect of $\alpha$-asarone was reversed. Hence, we could comment that nNOS/NO pathway involved in the anticonvulsant effect of $\alpha$-asarone, and NO played a biphasic role in PTZ modeling process, while iNOS was unrelated to the inhibition effect of $\alpha$-asarone on PTZ induced epileptiform activity. Apparently, further studies are needed to confirm these correlations. The relationship between eNOS and action mechanism of $\alpha$-asarone was unclear. Our findings may contribute to deeply understanding of the antiepileptic mechanisms of action of $\alpha$-asarone which are still unclear; and our findings reveal that drugs with properties of enhancement of nNOS/NO pathway might be a promising antiepileptic treatment, and also suggest that other drugs which can cause a change of NOS/NO pathway may influence the progression of epilepsy.

\section{Experimental procedures}

\subsection{Animals}

Fifty-four female Wistar rats weighing 210-260 g were experimented. They were raised standardly in laboratory, including a 12-h light/dark cycle, temperature control $\left(22 \pm 1^{\circ} \mathrm{C}\right)$ and standard feed, and rats were housed in groups of 3-4. All experimental protocols were performed in accordance with governmental approval according to local guidelines for the care and use of laboratory animals. Each experimental group was composed of six animals. Groups were assigned as following:

(1)sterile physiological saline solution + mixed solvent (DMSO/polyethylene glycol 300 1:1, v/v); (2)-asarone; (3) PTZ; (4) sterile physiological saline solution + mixed solvent (DMSO/polyethylene glycol 300 1:1, v/v) + PTZ; (5) aasarone + PTZ; (6) L-NAME + $\alpha$-asarone + PTZ; (7) 7-NI + $\alpha$ asarone + PTZ; (8) AG + $\alpha$-asarone + PTZ; (9) L-ARG + $\alpha$-asarone + PTZ.

\subsection{Operation}

The animals were anesthetized with urethane $(1.25 \mathrm{~g} / \mathrm{kg}$, i.p. $)$ and operations were performed in a stereotaxic apparatus (David Kopf, MI). After midline incision, two stainless steel screw electrodes (1 $\mathrm{mm}$ diameter) were placed over the bilateral frontal cortex(electrode coordinates: $2 \mathrm{~mm}$ lateral to sagittal suture and $1 \mathrm{~mm}$ anterior to bregma). Infiltration injection of procaine hydrochloride was given to 
minimize possible sources of pain during the entire surgery process. Rats were arranged to rest for one week or more time and to recover to health condition,

\subsection{Test compounds and dosing}

$\alpha$-Asarone and other test compounds applied in this work were bought from Sigma Chemical Co. $\alpha$ Asarone and 7-NI were dissolved in mixed solvent of dimethylsulfoxide with polyethylene glycol 300 (DMSO/polyethylene glycol $3001: 1$, v/v, respectively) and injected (i.p.) in a volume of $0.5 \mathrm{ml} / \mathrm{kg}$ body weight. L-NAME, PTZ, AG and L-ARG were dissolved in sterile physiological saline solution to such concentrations that requisite doses were injected intraperitoneally in a volume of $5 \mathrm{ml} / \mathrm{kg}$. $\alpha$ Asarone, at a dose of $80 \mathrm{mg} / \mathrm{kg}[42,43]$, was administered intraperitoneally $20 \mathrm{~min}$ before PTZ (50 $\mathrm{mg} / \mathrm{kg}$ i.p.) application in the aasarone + PTZ group. Animals received L-NAME $(60 \mathrm{mg} / \mathrm{kg}), 7-\mathrm{NI}(40$ $\mathrm{mg} / \mathrm{kg}), \mathrm{AG}(100 \mathrm{mg} / \mathrm{kg})$ and L-ARG $(500 \mathrm{mg} / \mathrm{kg}) 15 \mathrm{~min}$ [25,33] before $\alpha$-asarone (i.p.) application. The doses of drugs that were used in this study were chose according to previous published literatures $[20,25,33]$.

\subsection{Electrocorticographical recordings}

When recovering from the surgery, the rats were transferred to the observation cage. The rats were connected to a digital electroencephalogram (EEG) recording machine (32-channel, Nicolet Biomedical Inc., Middleton, WI) consciously by an isolated flexible cable. After the animals habituated to the experimental and recording conditions, the baseline EEG waves were recorded for $20 \mathrm{~min}$. Subsequently, chemicals were injected intraperitoneally as designed. The induced manifestation was noted and ECoG was recorded for $90 \mathrm{~min}$ after PTZ injection. The ECoG activity was continuously monitored using Nicolet 5.2. software program (Nicolet Biomedical Inc., Middleton, WI), with $70 \mathrm{~Hz}$ frequency, filtered at $0.01-1,500 \mathrm{~Hz}$ bandwidth and $0.03 \mathrm{~s}$ time constant 32-channel amplifier. A ground electrode was placed on tail of rats. The common reference electrode was fixed on right pinna and all electrodes were attached to a connecting socket. All recordings were saved on a computer. The frequency and amplitude of epileptiform discharge were visually analyzed in the off-line mode by our electroencephalographer (Dr. Su). Our electroencephalographer quantified ECoG clonic/min frequency, spike/min frequency, the highest microvolt voltage amplitude of clonic activity and spikes in every ten min during the whole range, and also distinguished true epileptiform ECoG spike signals from any artifact. In order to differentiate epileptiform waves from other nonepileptiform waves such as movement artifact, electroencephalographer monitored the whole recording and marked for artifact synchronously. Discharge amplitude was measured through the automated capture "Nicolet monitoring software" of the ECoG monitoring software.

Clonic attacks are seizures characterized by typical partial clonic activity influencing the face, head, vibrissae and forelimbs. Such clonic attacks are short, commonly lasting 1-2 s and can happen either individually or in multiple discrete episodes before generalization and over time, which are accompanied in the EEG with abnormal discharge, e.g. generalized and sharp polyspikes of various frequencies with shifting phase reversals (spike-wave or multispikes plus slow waves correlated with myoclonic jerks, which are feature of clonic attacks) [44]. Generalized convulsive attacks are performed by generalized whole-body clonus involving all four limbs and tail, rearing, wild running and jumping, sudden loss of upright posture and autonomic signs, such as hypersalivation and defecation, respectively. Generalized attacks were variable, and often followed by a quiescent period [44]. Spikes are defined as "paroxysmal, spontaneous, isolated, high-voltage, electrical discharge, characterized by an acute 
triangular form with a duration of less than $70 \mathrm{~ms}$, which could be clearly distinguished from background electroencephalography." [45].

\subsection{Data analysis}

The data are performed as the mean \pm S.E.M. Statistical analysis was complied by SPSS software (SPSS Science, V13.0, Chicago, IL, USA). Data was analyzed by one-way ANOVA and Bonferroniadjusted post-hoc t-tests for comparisons. And $\mathrm{p}<0.05$ was considered significant.

\section{Acknowledgement}

This work was supported by Youth Research Fund from the Second Affiliated Hospital of Dalian Medical University.

\section{References}

[1] P.K. Mukherjee, V. Kumar, M. Mal and P.J. Houghton, Acorus calamus: Scientific validation of ayurvedic tradition from natural resources, Pharm. Biol. 45 (2007), 1-16.

[2] H.S. Lee, Fungicidal property of active component derived from acorus gramineus rhizomeagainst phytopathogenic fungi, Bioresour. Technol. 98 (2007), 1324-1328.

[3] J. Cho, Y.H. Kim, J.Y. Kong, C.H. Yang and C.G. Park, Protection of cultured rat cortical neurons from excitotoxicity by asarone, a major essential oil component in the rhizomes of acorus gramineus, Life Sciences 71 (2002), 591-599.

[4] C. Huang, W.G. Li, X.B. Zhang, L. Wang, T.L. Xu, D.Z. Wu and Y. Li, Alpha-asarone from acorus gramineus alleviates epilepsy by modulating A-Type GABA receptors, Neuropharmacology 65 (2013), 1-11.

[5] B. Menon, K. Ramalingam and R.V. Kumar, Oxidative stress in patients with epilepsy is independent of antiepileptic drugs, Seizure 21 (2012), 780-784.

[6] H. Weisinger, Arginine metabolism and the synthesis of nitric oxide in the nervous system, Prog. Neurobiol. 64 (2001), 365-391.

[7] S. Moncada, R.M. Palmer and E.A. Higgs, Nitric oxide: Physiology, pathophysiology, and pharmacology, Pharmacol. Rev. 43 (1991), 109-142.

[8] V. Bashkatovaa, V. Narkevicha, G. Vitskovaa and A. Vanin, The influence of anticonvulsant and antioxidant drugs on nitric oxide level and lipid peroxidation in the rat brain during penthylenetetrazole-induced epileptiform model seizures, Prog. Neuropsychopharmacol. Biol. Psychiatry 27 (2003), 487-492.

[9] C.E. Elger, Epilepsy: Disease and model to study human brain function, Brain Pathol. 12 (2002), 193-198.

[10] V. Erakovic, G. Zupan, J. Varljen, J. Laginja and A. Simonic, Altered activities of rat brain metabolic enzymes in electroconvulsive shock-induced seizures, Epilepsia 42 (2001), 181-189.

[11] N. Patsoukis, G. Zervoudakis, C.D. Georgiou, F. Angelatou, N.A. Matsokis and N.T. Panagopoulos, Effect of pentylenetetrazole-induced epileptic seizure on thiol redox state in the mousecerebral cortex, Epilepsy Res. 62 (2004), 6574.

[12] S.A. Hamed and M.M. Abdellah, Trace elements and electrolytes homeostasis and their relation to antioxidant enzyme activity in brain hyperexcitability of epileptic patients, Journal of Pharmacological Sciences 96 (2004), 349-359.

[13] C. Iadecola, Bright and dark sides of NO in ischemic brain injury, Trends Neurosci. 20 (1997), 132-139.

[14] P. Tutka, M. Mlynarczyk, D. Zolkowska, Z. Kleinrok, M. Wielosz and S.J. Czuczwar, Nitric oxide and convulsions in 4-aminopyridine-treated mice, Eur. J. Pharmacol. 437 (2002), 47-53.

[15] S. Manikandan and R.S. Devi, Antioxidant property of asarone against noisestress-induced changes in different regions of rat brain, Pharmacol. Res. 52 (2005), 467-474.

[16] J.D. Sharma, P.C. Dandiya, R.M. Baxter and S.I. Kandel, Pharmacodynamical effects of asarone and beta-asarone, naturally occurring active substances, Nature 192 (1961), 1299-1300.

[17] P.C. Dandiya and Y.M. Chopra, CNS-active drugs from plant indigenous to india, Ind. J. Pharmacol. 2 (1970), 67-90. 
[18] Q.X. Chen, J.K. Miao, C. Li, X.W. Li, X.M. Wu and X.P. Zhang, Anticonvulsant activity of acute and chronic treatment with $\alpha$-asarone from acorus gramineus in seizure models, J. Biological \& Pharmaceutical Bulletin 36 (2013), 2330.

[19] L.F. Royes, M.R. Fighera, A.F. Furian, M.S. Oliveira, N.G. Fiorenza, J.C. Petry, R.C. Coelho and C.F. Mello, The role of nitric oxide on the convulsive behavior and oxidative stress induced by methylmalonate: An electroencephalographic and neurochemical study, Epilepsy Res. 73 (2007), 228-237.

[20] S.E. Smith, C.M. Man, P.K. Yip, E. Tang, A.G. Chapman and B.S. Meldrum, Anticonvulsant effects of 7-nitroindazole in rodents with reflex epilepsy may result from L-arginine accumulation or a reduction in nitric oxide or L-citrulline formation, Br. J. Pharmacol. 119 (1996), 165-173.

[21] H. Yasuda, M. Fuji, H. Fujisawa, H. Ito and M. Suzuki, Changes in nitric oxide synthesis and epileptic activity in the contralateral hippocampus of rats following intrahippocampal kainate injection, Epilepsia 42 (2001), 13-20.

[22] M. Yildirim, M. Ayyildiz and E. Agar, Endothelial nitric oxide synthase activity involves in the protective effect of ascorbic acid against penicillin-induced epileptiform activity, Seizure 19 (2010), 102-108.

[23] K.K. Borowicz, R. Starownik, Z. Kleinrok and S.J. Czuczwar, The influence of L-NGnitroargininemethyl ester, an inhibitor of nitric oxide synthase, upon the anticonvulsive activity of conventional antiepileptic drugs against maximal electroshock in mice, J. Neural Transm. 105 (1998), 1-12.

[24] N. Matsumura, K. Kikuchi-Utsumi and T. Nakaki, Activities of 7-nitroindazole and 1-(2-(trifluoromethylphenyl)imidazole independent of neuronal nitric-oxide synthase inhibition, J. Pharmacol. Exp. Ther. 325 (2008), 357-362.

[25] M. Ayyildiz, M. Yildirim and E. Agar, The involvement of nitric oxide in the anticonvulsant effects of alpha-tocopherol on penicillin-induced epileptiform activity in rats, Epilepsy Research 73 (2007), 166-172.

[26] H.S. Noh, D.W. Kim, G.J. Cho, W.S. Choi and S.S. Kang, Increased nitric oxide caused by the ketogenic diet reduces the onset time of kainic acid-induced seizures in ICR mice, Brain Res. 1075 (2006), 193-200.

[27] K.K. Akula, A. Dhir and S.K. Kulkarni, Nitric oxide signaling pathway in the anticonvulsant effect of adenosine against pentylenetetrazol-induced seizure threshold in mice, Eur. J. Pharmacol. 587 (2008), 129-134.

[28] P. Sardo, S. D’Agostino, V. Rizzo, F. Carletti, G. Lonobile and G. Ferraro, In the rat maximal dentate activation model of partial complex epilepsy, the anticonvulsant activity of levetiracetam is modulated by nitric oxide-active drugs, J. Neural Transm. 116 (2009), 831-839.

[29] G.B. De Sarro, E. Donato Di Paola, A. De Sarro and M.J. Vidal, L-Arginine potentiates excitatory amino acid-induced seizures elicited in the deep prepiriform cortex, Eur. J. Pharmacol. 230 (1993), 151-158.

[30] L. Sandirasegarane, J.R. Mikler, J.M. Tuchek and P.V. Sulakhe, Enhanced forebrain nitric oxide synthase activity in epileptic fowl, Brain Res. 735 (1996), 311-313.

[31] A.P. De Vasconcelos, C. Marescaux and A. Nehlig, Age-dependent regulation of seizure activity by NO in the developing rat, Brain Res. Dev. Brain Res. 107 (1998), 315-319.

[32] R.P. Smith, C.A. Louis, R. Kruszyna and H. Kruszyn, Acute neurotoxicity of sodium azide and nitric oxide, Fundam. Appl. Toxicol. 17 (1991), 120-127.

[33] M. Bosnak, M. Ayyildiz, M. Yildirim and E. Agar, The role of nitric oxide in the anticonvulsant effects of pyridoxine on penicillin-induced epileptiform activity in rats, Epilepsy. Res. 76 (2007), 49-59.

[34] A. Aslan, M. Yildirim, M. Ayyildiz, A. Güven and E. Agar, The role of nitricoxide in the inhibitory effect of ghrelin against penicillin-induced epileptiform activity in rat, Neuropeptides 43 (2009), 295-302.

[35] C. Rundfeldt, R. Koch, A. Richter, M. Mevissen, U. Gerecke and W. Löscher, Dosedependent anticonvulsant and proconvulsant effects of nitric oxide synthase inhibitors on seizure threshold in a cortical stimulation model in rats, Eur. J. Pharmacol. 274 (1995), 73-81.

[36] A.I. Prieto-Martín, S. Llorens, J.M. Pardal-Fernández, L.J. Muñoz, D.E. López, J. Escribano, E. Nava and C. de Cabo, Opposite caudal v-ersus rostral brain nitric oxide synthase response to generalized seizures in a novel ro-dent model of reflex epilepsy, Life Sciences 90 (2012), 531-537.

[37] L. D’Alessio, J. J. López-Costa, H. Konopka, D. Consalvo, E. Seoane, M.E. López, M.L. Guelman, S. Kochen and L.M. Zieher, NADPH diaphorase reactive neurons in temporallobe cortex of patients with intractableepilepsy and hippocampal sclerosis, Epilepsy Research 74 (2007), 228-231

[38] N. Kato, S. Sato, H. Yokoyama, K. Tayama and T. Yoshimura, Sequencial changes of nitric oxide levels in the temporal lobes of kainic acid-treated mice following application of nitric oxide synthase inhibitors and phenobarbital, Epilepsy Res. 65 (2005), 81-91.

[39] J.P. Leite, L. Chimelli, V.C. Terra-Bustamante, E.T. Costa, J.A. Assirati, G. de Nucci and A.R. Martins, Loss and sprouting of nitric oxide synthase neurons in the human epileptic hippocampus, Epilepsia. 43 (2002), 235-242.

[40] J. Garthwaite, Glutamate, nitric oxide and cell-cell signaling in the nervous system, Trends Neurosci. 14 (1991), 60-67.

[41] M. Robello, A.C. mico, G. Bucossi, A. Cupello, M.V. Rapallino and S. Thellung, Nitric oxide and GABAA receptor function in the rat cerebral cortex and cerebellar granule cells, Neuroscience 74 (1996), 99-105. 
[42] J.K. Miao, Q.X. Chen, X.M. Wu, C. Li and X.P. Zhang, Antiepile-ptic properties of alpha-asarone from acori graminei rhizoma in mice and rats seizuremodels, International Journal of Pharmacolog 8 (2012), 567-571.

[43] M. Cassani-Galindo, E. Madrigal-Budaijar, G. Chamorro, F. Diaz, J. Tamariz, J.J. Espinosa-Aguirre, In vitro genotoxic evaluation of three alpha-asarone analogues, Toxicol. In Vitro. 19 (2005), 547-52.

[44] M.S. Oliveira, A.F. Furian, L.F. Freire Royes, M.R. Fighera, N. G. Fiorenza, M. Castelli, P. Machado, D. Bohrer, M. Veiga, J. Ferreira, E.A. Cavalheiro and C.F. Mello, Cyclooxygenase-2/PGE2 pathway facilitates pentylenetetrazolinduced seizures, Epilepsy Research 79 (2008), 14-21.

[45] P. Gloor, Contributions of electroencephalography and electrocorticography to the neurosurgical treatment of the epilepsies, Adv. Neurol. 8 (1975), 59-105. 